See the related article beginning on page 497 .

\section{Insights into channel function via channel dysfunction}

\author{
John F. Leite, ${ }^{1}$ Nivalda Rodrigues-Pinguet, ${ }^{1,2}$ \\ and Henry A. Lester ${ }^{1}$
}

${ }^{1}$ Division of Biology, California Institute of Technology, Pasadena, California, USA ${ }^{2}$ Division of Biomedical Science, University of California Riverside, Riverside, California, USA

J. Clin. Invest. 111:436-437 (2003). doi:10.1172/JCI200317882.

The nicotinic synapse has been a touchstone for advances in neuroscience ever since Jean Nicot, the French ambassador to Portugal, sent some tobacco seeds home to Paris in 1550 with a note that the New World plant had interesting effects when smoked. Now the muscle nicotinic acetylcholine receptor ( $\mathrm{nAChR}$ ) is a well-studied example of ligand-gated ion channels. After a motor neuron is stimulated, the nerve impulse reaches the presynaptic terminal, where it evokes release of acetylcholine (ACh) into the synapse. The nAChR depolarizes the postsynaptic muscle and triggers muscle action potentials; muscle contraction follows. To date, several nAChR subtypes have been successfully isolated, purified, imaged, and expressed, and unitary currents have been recorded from these channels (1). Researchers continue to unravel the molecular mechanisms of these macromolecules that are embedded in membranes at vertebrate nerve-muscle synapses, at invertebrate nicotinic synapses (which explains why nicotine-producing tobacco plants have a select advantage against invertebrate pests), and in the vertebrate central

\footnotetext{
Address correspondence to: Henry A. Lester, Division of Biology, California Institute of Technology, 1200 East California Boulevard, Pasadena, California 91125, USA.

Phone: (626) 395- 4946; Fax: (626) 564-8709;

E-mail: lester@caltech.edu.

Conflict of interest: The authors have

declared that no conflict of interest exists. Nonstandard abbreviations used: nicotinic acetylcholine receptor ( $\mathrm{nAChR}$ ); acetylcholine (ACh); congenital myasthenic syndrome (CMS); cystine loop (cys-loop); acetylcholine binding protein (AChBP); slow-channel CMS (SCCMS); fast-channel CMS (FCCMS).
}

system (which explains Jean Nicot's fascination with those leaves). However, the precise structural events that trigger channel opening or "gating" remain mostly unknown.

Site-directed mutagenesis reveals some information about nAChR function but fails to give us an appreciation for the physiological significance of the receptor's biophysical properties. During the last decade, the venerable pharmacological approaches have been aided by newer insights from molecular genetics. We now appreciate that a number of naturally occurring mutations within nAChRs lead to severe
Figure 1 disorders. These ion-channel-associated disorders are collectively known as channelopathies. The nAChR is a heteropentamer assembled from $\alpha, \beta, \gamma$, (or $\varepsilon$ ) and $\delta$ subunits (Figure 1). Each subunit is thought to contain a large $\mathrm{N}$-terminal domain, followed by four transmembrane regions (M1-M4) with a large cytoplasmic loop between M3 and M4. In the central nervous system, at least five mutations linked to autosomal dominant nocturnal frontal lobe epilepsy lie within, or immediately adjacent to, M2 - the putative pore-forming region of neuronal nAChR subunits $(2,3)$. At the nerve-muscle synapse, at least 26 mutations in the extracellular, transmembrane, and cytoplasmic do-mains of the nAChR lead to different forms of congenital myasthenic syndrome (CMS) $(4,5)$. Thus these clinical cases aid in assigning physiological functions to the biophysical properties of nicotinic receptors.

In this issue of the JCI, Shen and colleagues report the channel kinetics from a valine to leucine mutation (V132L) located in the $\alpha$ subunit of the muscle $\mathrm{nAChR}$ within the signature cystine loop (cys-loop) (6). The cys-loop is a highly conserved structure found in

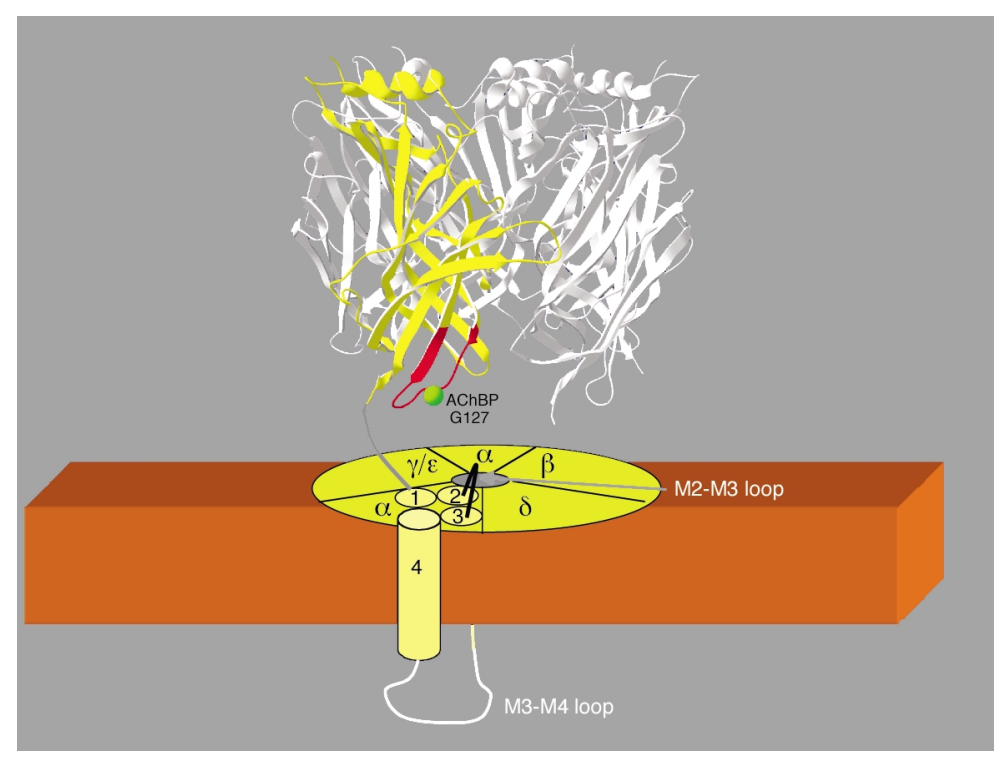

The structure of AChBP is illustrated over a representation of the transmembrane domain of $n A C h R$. Each subunit $(\alpha, \beta, \gamma$ or $\varepsilon, \delta)$ is labeled by its Greek symbol. The $\alpha$ subunit is shown in yellow, except for most of the signature cys-loop, which is shown in red, and AChBP G127 is shown in green (this residue aligns with $\mathrm{nAChR} \alpha \mathrm{V} 132$ ). The membrane spanning regions M1-M3 are represented on the bilayer surface, and M4 is also shown in cross section. 
every subunit of the ligand-gated ion channel superfamily. In a high-resolution structural model of the nAChR $\mathrm{N}$-terminus, based on the crystal structure of a snail (Lymnae stagnalis) acetylcholine-binding protein (AChBP), the cys-loop is located near the membrane surface (highlighted in red in Figure 1). Sequence analysis reveals that the AChR cys-loop contains a higher proportion of hydrophobic residues than the AChBP loop $(7,8)$. Furthermore, cryoelectron microscopy imaging of Torpedo electroplax membranes enriched with nAChR suggests that the cys-loop resides near the second (M2) and third (M3) transmembrane domains, where it may couple agonist binding to channel activation $(8,9)$.

The $\alpha V 132 \mathrm{~L}$ mutation leads to a severe form of postsynaptic CMS. Postsynaptic CMS mutations are classified as either slow- (SCCMS) or fast-channel congenital myasthenic syndromes (FCCMS), depending on their effects on the amplitude and waveform of postsynaptic currents at the muscle membrane. SCCMS mutations enhance agonist affinity or gating efficiency. FCCMS mutations reduce agonist affinity for the open channel or impair gating (4). In the present manuscript, the authors detail the detrimental effects of a FCCMS mutation on ACh binding and channel gating (6). Similar to other FCCMS mutations, $\alpha V 132 \mathrm{~L}$ attenuates the postsynaptic response to ACh. Specifically, $\alpha$ V132L reduces $A C h$ binding affinity for the closed channel state by 30 -fold. Consequently, ACh released into the synaptic space is predicted to activate only $10 \%$ of the available binding sites, much less than normal. Moreover, there is a fivefold decrease in the total ACh current due to an accelerated decay of the synaptic response. In short, receptors containing the $\alpha V 132 \mathrm{~L}$ mutation have pathologically little activation, and that for pathologically brief periods. This would be consistent with weak or nonexistent muscle stimulation in response to motor neuron activity.

\section{Do subunits with homologous sequences look alike?}

Interestingly, amino acid substitutions at positions corresponding to $\alpha \mathrm{V} 132 \mathrm{~L}$ in the $\beta, \varepsilon$, and $\delta$ subunits yielded significantly different phenotypes (6). Substitution of the aligning $V$ residue in the $\beta$ and $\varepsilon$ subunits produces virtually no effect, while substitution in the $\delta$ subunit produces a receptor with nearly the opposite phenotype of $\alpha V 132 L$. Specifically, $\delta V 134 \mathrm{~L}$ has slight effects on ACh binding affinity, but gating efficiency is reduced nearly fourfold. We suspect that each subunit's signature cys-loop folds and functions in a generally similar manner, but there is clearly some functional and struc-tural asymmetry at a more detailed level. Indeed, based on cryoelectron microscopy studies of the Torpedo $\mathrm{nAChR}$ in the open state (9), individual subunits do assume asymmetrical conformations and presumably have unique local environments. Apparently, even a small structural perturbation may lead to asymmetric changes in function.

\section{Implications from CMS studies}

Progress continues into the 21 st century. Shen and colleagues, including the Sine and Auerbach labs, are contributing to the study of the molecular pathophysiology of CMS. Furthermore, their biophysical analyses of the CMS mutations have provided us with some basic insights into the relationship between the structure and function of nAChRs. However, we still await refined structural information on the $\mathrm{nAChR}$ ion channel in order to further exploit present gating predictions based on similar kinetic studies.

1. Hille, B. 2001. Ion channels of excitable membranes 3rd edition. Sinauer Associates Inc. Sunderland, Massachusetts, USA. 814 pp.

2. Sutor, B., and Zolles, G. 2001. Neuronal nicotinic acetylcholine receptors and autosomal dominant nocturnal frontal lobe epilepsy: a critical review. Pflügers Arch. 442:642-651.

3. Bertrand, S., et al. 2002. ADNFLE mutations in CHRNA4 or CHRNB2 genes reveal critical positions of amino acid residues. Society for Neuroscience. 537:17. (Abstr.)

4. Engel, A.G., Ohno, K., and Sine, S. 2002. The spectrum of the congenital myasthenic syndromes. Mol. Neurobiol. 26:347-367.

5. Sine, S.M., et al. 2002. Naturally occurring mutations at the acetylcholine receptor binding site independently alter ACh binding and channel gating. J. Gen. Physiol. 120:483-496.

6. Shen, X.-M., et al. 2003. Mutation causing severe myasthenia reveals functional asymmetry of AChR signature cystine loops in agonist binding and gating. J. Clin. Invest. 111:497-505. doi:10.1172/JCI200316997.

7. Brejc, K., et al. Crystal structure of an ACh-binding protein reveals the ligand-binding domain of nicotinic receptors. Nature. 411:269-276.

8. Dougherty, D.A., and Lester, H.A. 2001. Snails, synapses and smokers. Nature. 411:252-253, 255

9. Unwin, N., Miyazawa, A., Li, J., and Fujiyoshi, Y. 2002. Activation of the nicotinic acetylcholine receptor involves a switch in conformation of the alpha subunits. J. Mol. Biol. 319:1165-1176. 